\title{
Detection and demodulation of non-cooperative burst signal
}

\author{
Feng Yue ${ }^{1}$, Wu Guangzhi ${ }^{1}$, Tao Min ${ }^{1}$ \\ ${ }^{1}$ China Satellite Maritime Tracking and Control Department, Jiangsu, Jiangyin, 214431, China
}

Keywords: satellite communication; non-cooperative communication; burst signal detection; matched filter; early phase estimate

\begin{abstract}
According to the typical three generations of maritime satellite communications frame format, the first use of effective detection of the burst signal energy detection algorithm combined with matched filter, then the frequency of the burst signal detection based on the results of estimation and phase estimation, then using the early phase locked loop injection combination achieved burst signal demodulation. Theoretical analysis and calculation simulation show that this method can adapt to the dynamic effects of low signal to noise ratio, the larger the frequency offset and downs, when the SNR is greater than $10 \mathrm{~dB}$, signal detection probability basically reached $100 \%$, the demodulation error rate of less than $2 * 10^{-3}$.
\end{abstract}

\section{Introduction}

Three Generations of the signal frame maritime mobile satellite communications applications / services such as short-term signal is often used in the form of sudden, for the detection of such signals can not be used to detect a continuous spectrum signal requires a signal from the time domain can accurately detect the signal appears time, otherwise it will affect the demodulation result of the signal. Carrier frequency and phase of these words or training sequence features not only can be used for signal detection, but also for the burst signal estimate. This field reconnaissance processing requirements combined with the fast frequency measurement and capturing an efficient way to achieve signal detection and demodulation. For these problems, requiring reconnaissance terminal to quickly and efficiently carry out the burst signal detection, quick-lock loop demodulator.

This article focuses on practical from an engineering point of view, for the detection of the burst signal energy detection method combined with matched filter of ways to enhance the probability of detection, the demodulated burst signal is first accurate estimate of the carrier frequency signal by means of a feed-forward manner, beginning with the aid of the carrier and a timing position, and then estimate the parameters of feed forward preset to the carrier synchronization loop and timing synchronization loop, so as to achieve fast carrier lock and symbol synchronization.

\section{Signal Detection}

Three Generations Mini-M standard maritime traffic signals subframe typical format shown in Figure 1, where the UW total of 84 bit, I and Q branches of the same 42 bit, are required to have a good cross-correlation properties. Traffic signals from a plurality of sub-burst frames.

\begin{tabular}{|c|c|c|}
\hline $\mathbf{U W}$ & Information & Redundancy \\
\hline $\mathbf{8 4}$ bit \\
Fig.1 Super frame of mini-M standard services signal
\end{tabular}

Signal detection is usually detected from the temporal and spectral characteristics of the signal characteristics, in some cases for some of the training sequence may also be used for testing. Based on the general characteristics of the frequency domain detection method is the frequency domain, time domain based on the general energy detection method, based on the training sequence is often belong to the matched filter. According to the characteristics of maritime satellite signals, we 
propose binding energy detection methods matched filtering the burst signal detection. Energy detection in the form of dual sliding window can quickly obtain the starting time of the burst, its block diagram shown in Figure 2, wherein the energy ratio curve $X(t)$ is the decision variable is the ratio of the energy of the window A B curve. Decision variable dual sliding window method of $X(t)$ and SNR values only, not to the signal energy, so you can set the threshold to achieve a relatively fixed burst signal detection. This algorithm is relatively simple, the key is to choose the length and every move is calculated once the good points of the window, the longer the window, the less points each movement is detected, the better the performance, at the expense of more computation. Meanwhile, in order to solve the broadband signal may exist in the multi-frequency signals can be combined with narrow-band digital filter mode signal detection.
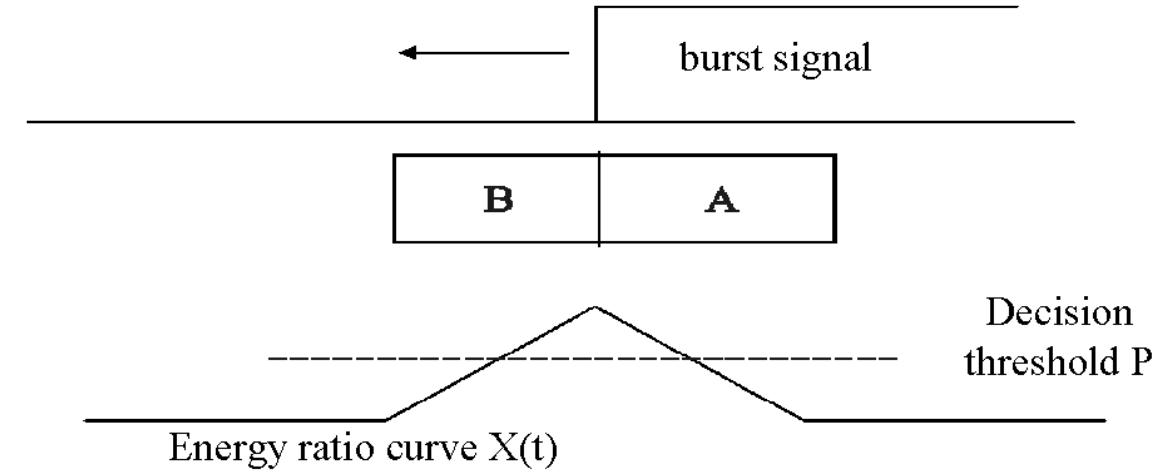

Fig.2 Detection response on double sliding windows

In addition, the signal detection matched filtering can be performed as follows. It assumed that burst contains features word baseband expression:

$$
S(t)=[I(\mathrm{t})+j Q(t)] e^{j\left(\Delta w t+\phi_{0}\right)}=z(t) e^{j\left(\Delta w t+\phi_{0}\right)}
$$

Results can be filtered by the filter after the match:

$$
R(\tau)=\int_{-\infty}^{+\infty} S(t) \odot h(\tau-t)^{*} d t=\int_{-\infty}^{+\infty} z(t) \odot h(\tau-t)^{*} e^{j\left(\Delta w t+\phi_{0}\right)} d t
$$

Requirements for signal detection matched filter relative deviation is not too large. Related peak characteristic features matched filter word closely related, when the feature-characteristic good even at low SNR can obtain high SNR correlation peak; extracting the sign bit before the match if the match filtering may filter to obtain a constant amplitude correlation peak, which can be achieved utilizing a fixed threshold to achieve a large dynamic signal detection.

Figure 3 (a) and (b) represent the burst signal to noise ratio for the case when the correlation peak header $10 \mathrm{~dB}$ and $0 \mathrm{~dB}$ without deviation obtained by the matched filter, to explain the situation to noise ratio is greater than 0 SNR little effect for the header.
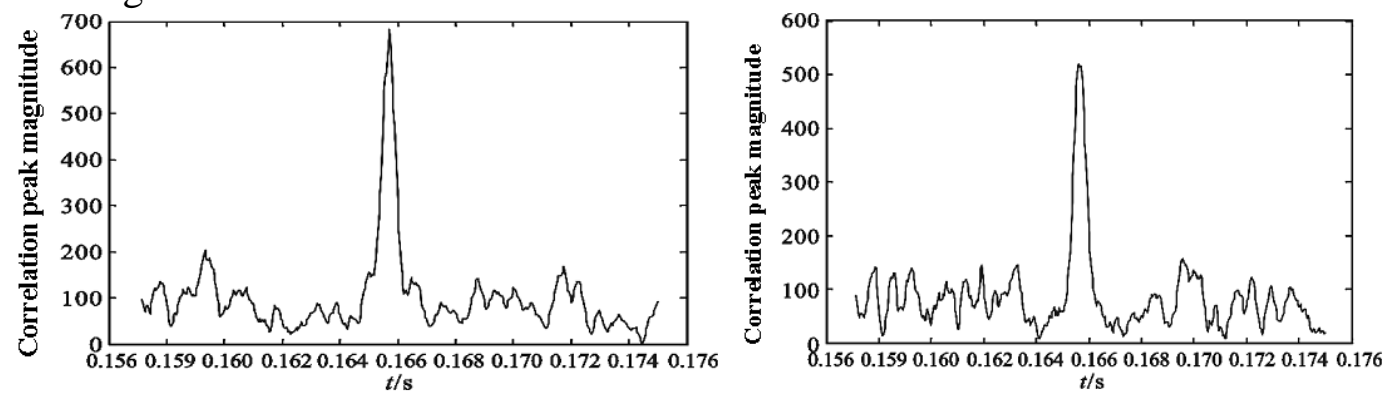

$\begin{array}{ll}\text { (a). Correlation peak signal to noise ratio of } 10 \mathrm{~dB} & \text { (b). } 0 \mathrm{~dB} \text { SNR correlation peak }\end{array}$

Fig. 3 Frame header correlation-peak in different SNR conditions

Figure 4 (a) and (b) represent the signal to noise ratio is $10 \mathrm{~dB}$, features characters period $\mathrm{T}$ is $0.008 \mathrm{~s}$, respectively, and offset $10 \mathrm{~Hz}$ and $50 \mathrm{~Hz}$ when obtained by the matched filter burst correlation peak header signal, the header described correlation peak for a large frequency offset influence. UW selected simulation parameters I and Q channel characteristic word 110110111110000011001100011101011010110011, shown it is the result of Gaussian white noise case of a single correlation peak. 


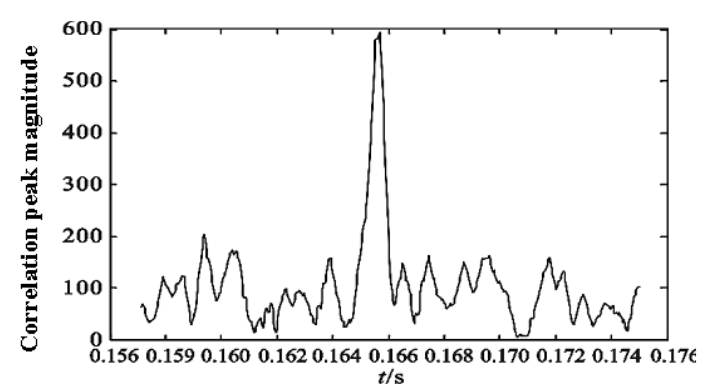

(a). An offset of $10 \mathrm{~Hz}$, the correlation peak

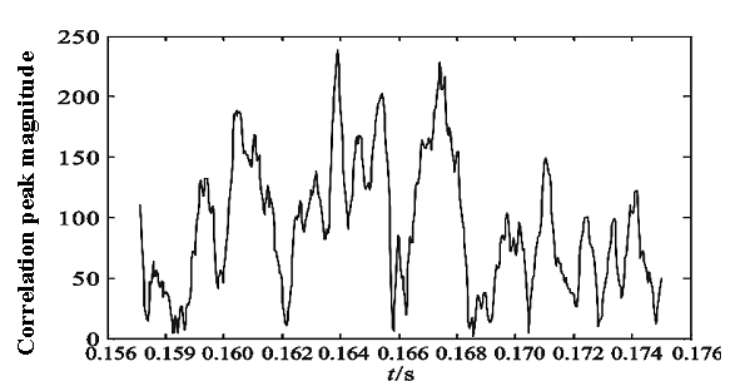

(b). Deviation is $50 \mathrm{~Hz}$ correlation peak

Fig. 4 Frame header correlation-peak in different frequency offset

Energy detection method, while simple, but in the actual electromagnetic environment factors susceptible to noise ratio detection precision, sudden strong interference and other factors, may increase certain false alarm and missed alarm probability, in order to enhance the detection performance at low SNR can [1] through literature detection algorithm based on short-term energy optimization of principal component analysis; matched filtering method works well in low SNR, high accuracy detection time, but easily offset by factors like, where you can the combination of two ways. Achieve energy detection preliminary testing, try to ensure that little leak alarm, then the trigger signal frequency measurement, and then matched filter for accurate signal detection threshold setting.

\section{Burst demodulation}

Satellite communications traffic signal usually burst communication mode, for non-cooperative signal demodulation such burst communication is difficult to achieve a simple feedback loop carrier and timing synchronization, which will inevitably result in the loss and destruction of business information and thus for demodulated burst signal needs frequency tracking speed, frequency measurement time is short, high precision, and the selected demodulation algorithm must rely on quickly to find a signal.

For the burst signal carrier and timing synchronization, usually with data aided (DA) and non-data-aided (NDA) in two ways. Auxiliary data transmission data using a known sequence to reach the front of the carrier frequency and phase estimate; NDA is based on data considered as random variables, to give the carrier frequency and phase by maximum likelihood estimation method.

After the matched filtering can be performed by the baseband signal, to obtain the maximum correlation peak phase values at a value that is the initial phase where the quasi-unique word signal sequence, and the initial phase value preset to the carrier synchronization loop, in order to ensure the carrier could have been synchronization status, so as to ensure the demodulation of zero lost work, the work diagram shown in Figure 5.

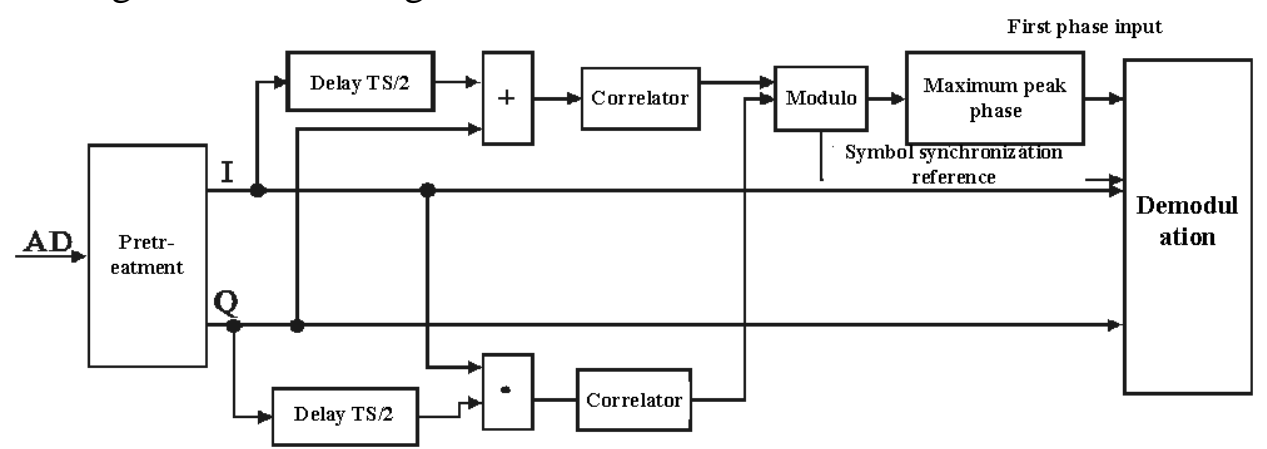

Fig.5 Demodulation diagram

AD IF sampling signal after pretreatment conversion, filtering, decimation and other digital obtained under I, Q baseband signals, due to the initial burst of maritime signal characteristics are known words, you can take advantage of the unique word signal detection and First phase strike. Signal detected by the I/Q correlators modulo get, I/Q correlators using sign before taking the 
symbols characteristic word correlation calculation, correlation peak threshold is characteristic word length, word of sampling points for each feature and the feature word autocorrelation peak can be set to a fixed value, can be adapted to more than $50 \mathrm{~dB}$ dynamic range requirements; deduced from the relevant formula I, Q correlation peak maximum road is OQPSK modulation of the initial phase, by the early phase preset to conventional OQPSK carrier synchronization loop mode carrier loop to achieve fast lock; by the maximum value of the correlation peak position can be obtained the optimal sampling point symbol synchronization position, which can achieve zero loss of useful information OQPSK signal burst demodulation purposes.

\section{Test case verification}

Figure 6 shows a modulation signal for maritime emergencies OQPSK whether to adopt the solution estimate carrier frequency and phase synchronous demodulation constellation preset loop carrier under contrast diagram in which (a) is not properly represented by the preset initial phase of tune constellation constellation difficult convergence, demodulation error vector magnitude (EVM) is poor; (b) shows the correct preset initial phase demodulation constellation, the constellation converge well, and from the burst signal constellation at the beginning had converged.

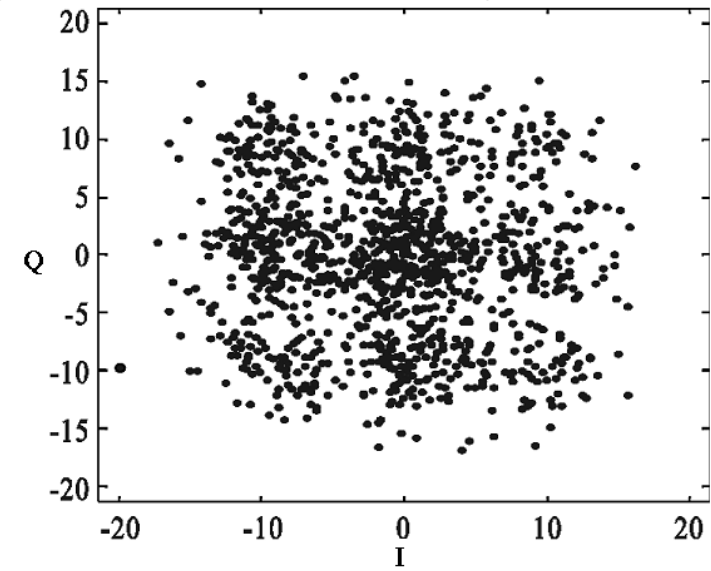

(a). The initial phase error demodulation constellation

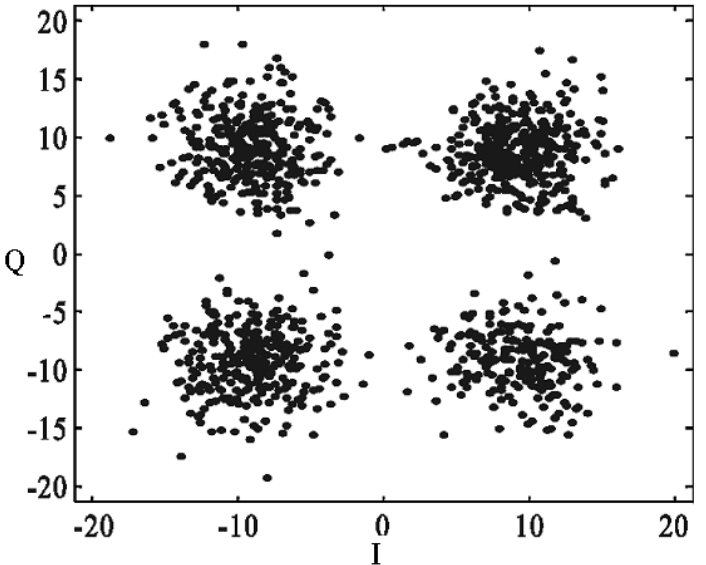

(b). demodulation initial phase correct constellation

Fig. 6 Performance contrast of OQPSK demodulator

For maritime auxiliary data signal such short bursts, by validating simulation and signal detection method using a power quickly burst signal detection, signal to noise ratio at $7 \mathrm{~dB}$ can be achieved when the signal detection probability of $95 \%$, detection probability of $100 \%$ to reach 10 $\mathrm{dB}$ SNR; the use of a matched filter method, in $5 \mathrm{~dB}$ SNR can reach more than $99 \%$ probability of detection. In order to enhance the demodulation performance, increase the use of features characters accurately estimate the carrier frequency offset, so that the algorithm is further optimized.

\section{Conclusion}

For non-cooperative analysis of maritime satellite communications, burst signal, the most important is to realize the burst signal detection and demodulation before subsequent analysis. For the burst signal detection and demodulation can draw some general analytical methods, but also need to use some prior information signal in order to enhance the analysis of the data assist performance. The proposed combination of a number of ways to detect and zero loss burst signal demodulation scheme has a certain universality, for a similar signal format signal analysis to provide certain engineering reference, able to adapt to a low signal to noise ratio, the more large signal dynamic and frequency offset. If there is no word for the feature or features not known in advance of the burst of the word, if you need to adapt to the low signal detection and zero loss of signal demodulation a relatively large degree of difficulty, can further study. 


\section{References}

[1] SUI Dan,GE Lindong,QU Dan. A novel presence detection algorithm based on the energy detection for burst signals[J]. Signal Processing,2008,24 (4):614 - 617.(in Chinese)

[2] SUI Dan. Study on Blind Detection Technologies of Communication Signals[D]. Zhengzhou:PLA Information Engineering University,2008. (in Chinese)

[3] ZENG Xingwen,GUO Feng,LIU Naian,et al. Influence of freqency offset on SAWTDL correlate characteristics[J]. Jounal of Xidian University,2008,25 (3):290 -293. (in Chinese)

[4] Mengali U,Andrea A. Synchronization techniques for digitalreceivers[M]. New York:[s. n.],2007. 